\title{
Dietary Probiotics Alter Broiler Intestinal Permeability Parameters but not Performance
}

\begin{abstract}
DOI:10.31274/air.11541
Meaghan Meyer, Graduate Research Assistant, Department of Animal Science; Elizabeth Bobeck, Assistant Professor, Department of Animal Science, Iowa State University

Summary and Implications

Since the Veterinary Feed Directive became effective in 2015, poultry producers have sought a replacement for antibiotics in commercial broiler diets to promote growth and gut health. A series of poultry feed additives including probiotics, prebiotics, yeast products, etc., have shown some success in improving growth performance of broilers. Two solid state fermentation directfed microbial (DFM) products from two different lactobacillus strains were fed at two inclusion levels $(0.05$ and $0.1 \%$ ) to study the effects on weight gain, FCR, and $\mathrm{ADG}$, and gut health parameters following feed restriction and FITC-dextran (FITC-d) gavage. Under our research conditions, certain dietary treatments decreased "gut leakage" as measured by increased FITC-d fluorescence in the serum as a result of feed restriction, but performance parameters were not significantly affected by dietary
\end{abstract} treatments.

\section{Introduction}

Commercial broiler producers are continuously looking for ways to improve feed efficiency and growth in their flocks and are turning more and more to feed additives to accomplish this. Probiotic additives have shown some capability to do this, presumably due to their ability to promote healthy bacterial colonization and general gut health. FITC-d gavage is a well-published gut integrity parameter used to quantify "gut leakiness". Dextran is a large molecule that is not typically absorbed from the digesta across the intestine into the bloodstream; however, if the gut is challenged by stress or damage and tight junction integrity is reduced, dextran will translocate into the bloodstream. FITC tagging of dextran allows for quantification of this absorbed dextran in serum pulled from blood drawn one hour after FITC-d gavage. Additionally, feed restriction is known to cause stress in broilers that triggers this translocation. Thus, the objectives of this work were to determine if probiotics improved growth and feed efficiency, and decreased "gut leakage" during feed restriction.

\begin{abstract}
Materials and Methods
Four hundred and eighty Ross 708 broilers were obtained from a commercial hatchery, transported to the Iowa State Poultry Research and Teaching Farm on day of hatch, and raised for a 6-week grow-out period. The birds were housed on re-used litter (fourth time reused) in forty pens of 12, with ad libitum access to feed and water. Basal starter, grower, and finisher diets were formulated according to Ross 708 production guidelines. Five treatment groups were assigned using two different probiotic strains; $0.05 \%$ Probiotic Diet (PD) 1, 0.10\% PD1, 0.05\% PD2, 0.10\% PD 2 , and control (CON), a basal diet with no probiotic inclusion. Each diet was randomly assigned to eight pens. The birds were fed a starter diet for weeks 1-2, grower for weeks 3-4, and finisher weeks 5-6. Birds were weighed upon placement (day 0 ) and upon conclusion of each 2week performance period. Feed disappearance was recorded throughout.

On day 26 , a subset of 20 out of 40 pens were weighed, and half were subjected to a 12-hour feed restriction (10 FR pens, 10 not-FR). On day 27, a FITC-d oral gavage was administered to six birds/pen in ten pens including 2 pens/treatment, (60 birds, five FR pens, five not-FR) at an inclusion of $8.32 \mathrm{mg} / \mathrm{kg}$ based on individual bird weights taken the day before. Blood samples were drawn from the 5 FR+FITC-d pens ( 6 birds/pen), 5 not-FR+FITC-d pens ( 6 birds/pen), and additionally, 5 FR non-FITC-d pens ( 5 birds/pen), and 5 not-FR non-FITC-d pens ( 5 birds/pen) to use as negative controls/blanks.
\end{abstract}

Serum was separated and stored at $-20^{\circ} \mathrm{C}$ until samples were thawed, diluted (1:5 in saline), plated in duplicate in black 96-well plates, and read at 485/528 nm excitation and emission wavelengths, respectively. Data were analyzed using PROC t-test (FR and not-FR values within each treatment) and PROC mixed (comparisons of all treatments) with differences detected using PDIFF, on SAS version 9.4.

\section{Results and Discussion}

Across all performance parameters (feed intake, weight gain, FCR, and ADG), there were no significant differences detected between treatments for the main effect of PD, inclusion rate, or the interaction between PD and inclusion rate $(\mathrm{P}>0.05$; Table 1). The lack of effect of probiotic treatments in performance may have been due to the clean research environment as compared to commercial barns. Although the litter had been re-used 4 times to better reflect a commercial environment, the research setting does not contain pathogens typical to many commercial farm settings.

Copyright $(\mathcal{C} 2020$ by the Authors. This is an open access article published under the CC BY-NC license (https://creativecommons.org/licenses/by-nc/4.0/), which allows for non-commercial reuse with proper attribution. 
The FITC-d relative fluorescence serum data indicated that the 12-hour feed restriction was successful, as more FITC-d was found in the serum of FR vs. control nonfeed restricted birds $(\mathrm{P}<0.0001$, Figure 1$)$. The main effect of $\mathrm{PD}$ alone was also significant $(\mathrm{P}=0.0018)$, with $0.05 \% \mathrm{PD} 2$ resulting in the greatest amount of FITC-d crossing into circulation, hence the diet by restriction interaction was also significant $(\mathrm{P}<0.0001)$. No treatments were significantly different after feed restriction apart from $0.05 \%$ PD2 $(\mathrm{P}<0.0001)$, suggesting a benefit from both $0.05 \%$ and $0.10 \%$ PD1 and only $0.10 \%$ PD2 in maintaining intestinal barrier function when birds were subjected to significant stress (FR).

When comparing non-FR to FR within diet only, FR again successfully stressed the gut by increasing fluorescence numerically in every treatment group compared to birds non-FR within in the same treatment using a t-test (Table 2). This increase in "gut leakiness" was significant in the CON $(\mathrm{P}=0.0004), 0.05 \% \mathrm{PD} 1(\mathrm{P}=0.0035)$, and $0.05 \% \mathrm{PD} 2(\mathrm{P}=0.0018)$ treatments when compared within each diet only (+FR / -FR). There was not a significant increase in FITC-d appearance in circulation in the $0.10 \% \mathrm{PD} 1(\mathrm{P}=0.2818)$ and $0.10 \% \mathrm{PD} 2(\mathrm{P}=0.6078)$ treatments. According to these data, the $0.10 \%$ inclusion rate prevented gut leakage during a FR challenge.

Although the performance data were not affected by treatment, the probiotic inclusions clearly had a positive effect on gut integrity, with the smallest percent increases in FITC-d absorbance seen in the $0.10 \%$ inclusion rates of PD1 and PD2. The $0.05 \%$ PD2 fluorescence readings showed the greatest amount of variation and the greatest percent increase, meaning the gut integrity of those birds was the most compromised by the FR challenge. This treatment group had, numerically, the lowest overall feed intake of all diets, thus decreased consumption of the probiotic. This may be interpreted as a cause for negative effect on gut health. It is unclear why this specific inclusion level/treatment showed the lowest intake and weight gain; further studies to determine if this is a possible unintended outcome of this specific additive are warranted.

Under our research conditions, two probiotic feed additives fed to Ross 708 broilers at $0.05 \%$ and $0.10 \%$ each did not affect feed conversion nor weight gain but were able to maintain gut integrity following a 12-hour feed restriction in the $0.10 \%$ inclusion treatment groups of both additives. Future work involving pathogenic intestinal challenge may provide insight into mechanisms of improving integrity due to inclusion of probiotics.

\section{Acknowledgements}

The authors would like to thank the Poultry Research and Teaching Farm staff for help in animal husbandry, barn management, and collection of performance measures, as well as undergraduate student Caitlyn Spencer for help onfarm. 
Table 1. Average intake, gains, $\mathrm{ADG}^{1}$, and $\mathrm{FCR}^{2}$ by each 2-week performance period and overall, averaged per bird

\begin{tabular}{|c|c|c|c|c|c|c|c|}
\hline $\begin{array}{c}\text { Performance } \\
\text { Measure }\end{array}$ & CONTROL & $\begin{array}{l}\text { PD1 }^{3} \\
0.05 \%\end{array}$ & $\begin{array}{c}\text { PD1 } \\
0.10 \%\end{array}$ & $\begin{array}{l}\text { PD2 }^{4} \\
0.05 \%\end{array}$ & $\begin{array}{c}\text { PD2 } \\
0.10 \%\end{array}$ & Pooled SEM & P-value \\
\hline \multicolumn{8}{|l|}{ Feed intake (kg) } \\
\hline Starter & 0.41 & 0.40 & 0.40 & 0.40 & 0.40 & 0.011 & 0.876 \\
\hline Grower & 1.33 & 1.22 & 1.25 & 1.23 & 1.33 & 0.038 & 0.106 \\
\hline Finisher & 2.27 & 2.23 & 2.22 & 2.18 & 2.41 & 0.083 & 0.350 \\
\hline Overall & 4.01 & 3.85 & 3.87 & 3.81 & 4.14 & 0.115 & 0.242 \\
\hline \multicolumn{8}{|l|}{ Weight gain (kg) } \\
\hline Starter & 0.28 & 0.29 & 0.28 & 0.29 & 0.28 & 0.008 & 0.744 \\
\hline Grower & 0.83 & 0.81 & 0.82 & 0.84 & 0.83 & 0.021 & 0.954 \\
\hline Finisher & 1.34 & 1.35 & 1.31 & 1.27 & 1.42 & 0.051 & 0.352 \\
\hline Overall & 2.45 & 2.45 & 2.41 & 2.40 & 2.53 & 0.069 & 0.702 \\
\hline \multicolumn{8}{|l|}{$F C R$} \\
\hline Starter & 1.38 & 1.42 & 1.45 & 1.42 & 1.47 & 0.046 & 0.740 \\
\hline Grower & 1.58 & 1.49 & 1.54 & 1.51 & 1.62 & 0.052 & 0.407 \\
\hline Finisher & 1.73 & 1.67 & 1.67 & 1.78 & 1.78 & 0.080 & 0.736 \\
\hline Overall & 1.63 & 1.58 & 1.60 & 1.60 & 1.70 & 0.053 & 0.585 \\
\hline \multicolumn{8}{|l|}{$A D G(\mathrm{~kg})$} \\
\hline Starter ADG & 0.02 & 0.02 & 0.02 & 0.02 & 0.02 & 0.001 & 0.744 \\
\hline Grower ADG & 0.06 & 0.06 & 0.06 & 0.06 & 0.06 & 0.002 & 0.954 \\
\hline Finisher ADG & 0.10 & 0.010 & 0.09 & 0.09 & 0.10 & 0.004 & 0.352 \\
\hline Overall ADG & 0.06 & 0.06 & 0.06 & 0.06 & 0.06 & 0.002 & 0.702 \\
\hline
\end{tabular}

${ }^{1} \mathrm{ADG}=$ Average daily gain

${ }^{2} \mathrm{FCR}=$ Feed conversion ratio

${ }^{3} \mathrm{PD} 1=$ Probiotic Diet 1

${ }^{4} \mathrm{PD} 2=$ Probiotic Diet 2 
Figure 1. Main effect of feed restriction; values are expressed as mean fluorescence $(\mathrm{ng} / \mathrm{mL})$ of serum fluorescein isothiocyanate dextran (FITC-d) in 12-hour feed restriction model of broiler chickens. Comparisons made between all diets used Proc mixed on SAS 9.4. *Different letters denote means are significantly different $(P \leq 0.05)$.

\section{Main Effect Feed Restriction}

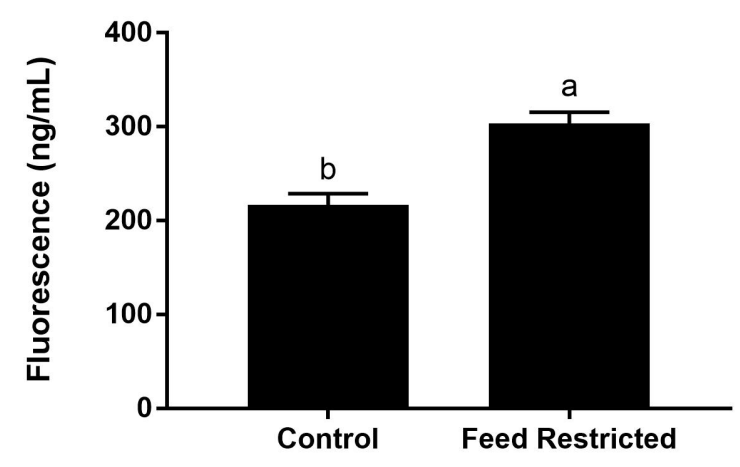

Table 2. Fluorescence readings of serum fluorescein isothiocyanate dextran (FITC-d) in 12-hour feed restriction model on different diet treatments of broiler chickens; direct comparison only within diet

*P-values presented are t-test results comparing feed restriction and control fluorescence means within each diet, $\mathrm{P}<0.05$

\begin{tabular}{lclccc}
\hline Experimental group & $\begin{array}{c}\text { Mean fluorescence } \\
(\mathbf{n g} / \mathbf{m l})\end{array}$ & SEM & $\begin{array}{c}\text { Increase in } \\
\text { fluorescence due } \\
\text { to FR (ng/ml) }\end{array}$ & \% Increase & P-value* \\
\hline Control + FR ${ }^{1}$ & 282.9 & 12.590 & & & \\
Control - FR & 220.7 & 4.383 & 62.2 & $28.2 \%$ & $0.0004^{*}$ \\
\hline $0.05 \%$ PD1 + FR & 262.5 & 9.426 & & & \\
$0.05 \%$ PD1 - FR & 220.9 & 8.537 & 41.6 & $18.8 \%$ & $0.0035^{*}$ \\
$0.1 \%$ PD1 + FR & 256.8 & 23.160 & & & \\
$0.1 \%$ PD1 - FR & 224.4 & 17.947 & 32.4 & $14.4 \%$ & 0.2818 \\
\hline $0.05 \%$ PD2 2 FR & 471.1 & 68.032 & & & \\
$0.05 \%$ PD2 - FR & 192.7 & 8.576 & 278.4 & $144.5 \%$ & $0.0018^{*}$ \\
$0.1 \%$ PD2 + FR & 244.4 & 27.476 & & & \\
$0.1 \%$ PD2 - FR & 225.9 & 22.409 & 18.5 & $8.2 \%$ & 0.6078 \\
\hline
\end{tabular}

${ }^{1} \mathrm{FR}=$ Feed restriction

${ }^{2} \mathrm{PD} 1=$ Probiotic Diet 1

${ }^{3} \mathrm{PD} 2=$ Probiotic Diet 2 